\title{
Memoria y tiranía en la Confederación Aquea helenística (s. III-II a.C.)
}

\author{
Álvaro M. Moreno Leoni \\ Universidad Nacional de Córdoba (Rep. Argentina) \\ moreno.leoni@gmail.com \\ Memory and Tyranny in the Hellenistic Achaean Confederacy \\ (III-II Centuries BC)
}

El otorgamiento de amnistías a varios tiranos peloponesios durante la expansión de la Confederación Aquea helenística no ha sido estudiado. En este artículo se propone analizar algunos aspectos políticos y culturales de esta importante práctica tomando en cuenta los desarrollos teóricos e historiográficos recientes en el campo de estudios sobre memoria colectiva e identidad. En ese sentido, se explorará aquí el alcance de la amnistía garantizada a estos regímenes tiránicos, enfocando en cómo los miembros de la élite política aquea eligieron recordar y no olvidar a los tiranos y construir una visión colectiva y compartida del pasado en la cual la lucha contra ellos se volvió una piedra angular para el proceso de definición de su identidad.

Palabras clave: Confederación Aquea; tiranía; memoria colectiva.
The grant of amnesties to several Peloponnesian tyrants during the expansion of the Hellenistic Achaean Confederacy has not been studied. In this paper it is proposed to analyze some political and cultural features of this main practice taking into account the recent theoretical and historiographical developments on the field of collective memory and identity studies. In this sense it will be explored here the scope of the amnesty granted to these tyrannical regimes, focusing on how the members of the Achaean political elite chose to remember and not forget the tyrants and to build a collective and shared vision of the past in which the fight against them became a cornerstone to their identity definition process.

Key words: Achaean Confederacy; tyranny; collective memory.

\section{INTRODUCCIÓN}

La Confederación Aquea fue una de las experiencias estatales helenísticas más significativas $\mathrm{y}$, como tal, ha llamado la atención de historiadores desde el siglo XIX (Freeman 1863; Dubois 1885; Aymard 1970; Bastini 1987; Didu 1993; Nottmeyer 1995). Su rápida expansión por el Peloponeso es entendible 
cuando se tiene en cuenta la centralidad de la sympoliteía. La construcción de una doble ciudadanía, políada y suprapolíada, permitió a algunas pequeñas póleis del norte del Peloponeso plasmar una forma estatal federal a partir del 281/0 a.C. y superar para mediados de ese siglo los estrechos límites étnicoregionales de Acaya y lograr a comienzos del siglo siguiente la unidad peninsular. Existen algunos estudios sobre los aspectos jurídicos de esta expansión, así como también sobre algunos de sus rasgos políticos, fundamentalmente, sobre la conformación de una élite política federal ${ }^{1}$. Se han realizado algunas investigaciones con foco en las vidas, ideas y acciones de los principales líderes aqueos, como Arato y Filopemén, quienes han sido considerados como los grandes forjadores de la Confederación Aquea (Ferrabino 1972; Walbank 1933; Errington 1969; Golan 1973, 1974). Entre las décadas del '80 y '90 del siglo pasado se publicaron también algunas monografías generales que, desde una perspectiva de la historia política, se plantearon como objetivo la reconstrucción de los acontecimientos de alguna de las etapas históricas aqueas (Aymard 1970; Bastini 1987; Didu 1993; Nottmeyer 1995).

No se ha publicado en los últimos veinte años, sin embargo, ninguna obra de interpretación general sobre la Confederación Aquea, lo que sí ha ocurrido, en cambio, en el caso de los etolios (Scholten 2000; Grainger 1999). Existe en la actualidad una gran necesidad de una obra de similares características que brinde una visión sintética y de conjunto, en sintonía con una tendencia detectable en los últimos años hacia el estudio de aspectos político-culturales de la experiencia histórica aquea. Así, por ejemplo, en un trabajo anterior (Moreno Leoni 2013) hemos indagado sobre la progresiva articulación de un discurso que identificaba el espacio geográfico peloponesio con el territorio federal, permitiendo a los aqueos imaginar la unidad peninsular a partir del último cuarto del s. III a.C. Koehn (2007, pp. 135-155), por su parte, ha explorado cómo el discurso de la lucha contra las tiranías se convirtió en un instrumento propagandístico que permitió a los aqueos, como poder de mediano tamaño, legitimar ante la opinión pública griega su expansión territorial.

Ambos trabajos han mostrado cómo el discurso de «liberación» del Peloponeso de manos de los tiranos, y su correlato de la «unificación» peninsular,

\footnotetext{
${ }^{1}$ Algunos estudios sobre el desarrollo de la ciudadanía federal: Larsen 1968; Lozano 2005; Beck 1997; Lehmann 2001. Una excelente discusión sobre los aspectos jurídicos: Pascual 2007. Sobre la conformación de una élite política federal aquea: O’Neil 1984/6; «classe de politiciens»: Aymard 1938, p. 328.
} 
habrían legitimado, y simultáneamente alimentado, los objetivos aqueos de expansión. Al mismo tiempo, han puesto de relieve que no se trató de un discurso acabado, sino en proceso de constante redefinición en el marco de las distintas campañas contra los regímenes tiránicos de la península. A partir de esto se abren una serie de interrogantes a los que buscamos dar respuesta aquí: ¿Qué consecuencias tuvo la construcción de este discurso para la cohesión y la identidad de la élite política federal? ¿De qué modo su estructuración contribuyó a delinear una «percepción compartida» del pasado entre los aqueos? ¿En qué medida coadyuvó a la construcción de una «memoria colectiva»? Finalmente, ¿qué lugar jugó el recuerdo de las tiranías en esta visión selectiva del pasado?

Nuestro problema de estudio se enmarca dentro de debates actuales dentro de la historiografía. Los problemas ligados a la memoria y a la conformación de las identidades en las sociedades en el pasado se han convertido, al menos desde hace unos treinta años, en un campo de estudio central para los historiadores que han reconocido la importancia de las actitudes y reacciones de las sociedades contemporáneas, que han atravesado procesos históricos traumáticos, hacia su pasado. Este interés también es reconocible en el campo de la historia griega antigua donde, desde mediados de los '90 del siglo pasado, algunos trabajos se han concentrado en el fenómeno de la memoria histórica de acontecimientos traumáticos recientes. Se ha prestado particular atención a la memoria de guerras, represiones y conflictos civiles del pasado inmediato, estudiando la variedad de respuestas que las generaciones siguientes ensayaron para elaborar el recuerdo de los mismos (Loraux 2008; Wolpert 2002; Flower 2006, pp. 17-41; Savalli-Lestrade 2009). Ha habido también estudios dentro de un plano de larga duración histórica, más específicamente, apuntando a procesos vinculados a la memoria histórico-cultural (Lafond 2006; Luraghi 2008). En los últimos años, se ha sumado finalmente una preocupación por el problema de los «lugares de memoria» de acuerdo con la propuesta de Pierre Nora (Gangloff 2013; Haake y Jung 2011).

El sustento teórico de estos estudios es la clásica premisa sobre memoria colectiva de Halbwachs (2004 [1950], pp. 105-138), quien consideraba que la memoria sólo era pensable como una «reconstrucción» colectiva de los recuerdos a partir de los «marcos sociales» del presente de los actores. Esta «reconstrucción» se observa como una operación compleja, de creación cultural y consumo, en la que se produce una interacción y también una negociación en torno a las tradiciones culturales entre los hacedores de memoria 
y sus consumidores, que interactúan en el marco de una arena política competitiva (Kansteiner 2002, p. 179). Es decir, la memoria lejos de ser un fenómeno estrictamente psicológico, es un fenómeno social que se define y redefine dentro de un marco de acción política sobre la base de los criterios de utilidad y credibilidad (Luraghi 2008, p. 13). En los últimos años, se ha puesto el acento además en la importancia de los procesos concretos de recepción de la memoria, así como de su significación (Kansteiner 2002, pp. 190-195; Ober 2003).

Partiendo de estas herramientas teóricas, se pretende aquí contribuir a la comprensión de los procesos de construcción de memoria entre los miembros de la élite política aquea entre los s. III-II a.C. En particular, se busca indagar sobre los mecanismos de construcción de la memoria de la tiranía, poniendo el acento en el otorgamiento de amnistías a los tiranos y en las actitudes que las mismas generaron de cara al olvido de estos regímenes, prestando particular atención al abuso político de la memoria (Ricoeur 2004, pp. 82-83). En el marco de este problema, se examinará también el tratamiento que Polibio hizo de la ejecución de Aristómaco, ex-tirano de Argos, intentando reconocer en qué medida puede considerarse a este historiador como un caso de recepción, selectiva y orientada desde el presente, de esta memoria de las tiranías construida en el interior de la élite política aquea. Finalmente se propondrá a modo de epílogo una reflexión sobre los límites políticos de la memoria de los regímenes tiránicos en el marco de las guerras contra Esparta.

\section{LAS TIRANÍAS PELOPONESIAS: ENTRE LA AMNISTÍA Y LA EXPANSIÓN AQUEAS}

Plutarco critica por varias razones la actuación de Arato durante la Guerra Cleoménica (227-222 a.C.), pero, principalmente, por su petición de ayuda a Antígono III Dosón, pues para él Arato habría con ello traicionado la esperanza de una Grecia unida e independiente. En el marco de esta crítica, probablemente inspirada por su fuente, es decir, por Filarco, Plutarco nos ha dejado quizá una huella importante para entender el problema de la memoria de la tiranía entre los aqueos. Dice el biógrafo que Arato era vivamente criticado por sus conciudadanos por la sumisión en la que había colocado nuevamente a los aqueos frente a los macedonios, pero que, por su parte, este se sentía particularmente apesadumbrado por el destino que habían sufrido unas estatuas. Antígono, estando en Argos, había ordenado derribar las representaciones de los héroes aqueos que habían tomado parte junto con 
Arato en la captura del Acrocorinto (243 a.C.), aunque se había respetado la estatua del líder sicionio, y que, en su lugar, había hecho nuevamente levantar las de los tiranos argivos (Plu., Arat. 45.3). Estos últimos habían sido aliados tradicionales de Macedonia ${ }^{2}$. Por un lado, Arato estaba molesto, precisamente porque él había hecho destruir sistemáticamente las imágenes de los tiranos en las póleis incorporadas a la Confederación, como había ocurrido en Sición

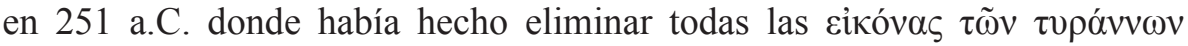
(«las imágenes de los tiranos») (Plu., Arat. 13.1). Por otro lado, parece haber sido una práctica habitual en el siglo III a.C., como muestra la ley de Ilión (280 a.C.), que establecía la destrucción de los monumentos erigidos por los culpables de un régimen tiránico en el marco de su abolitio memoriae ${ }^{3}$, o las medidas tomadas por Atenas en contra de la memoria de Filipo V, tras «tiranizarlo»y asimilarlo a los pisistrátidas $(200 \text { a. C. })^{4}$.

La construcción de un monumento, como es el caso de un grupo estatuario, no se corresponde en un sentido estricto con el recuerdo de los actores de la gesta representados, sino, más bien, con el escenario político y el marco social desde el cual se decide recordar y, por lo tanto, reinterpretar las personas o sus acciones desde el presente (Jelin y Langland 2003, p. 9). Desconocemos la inscripción que acompañaba a estas estatuas, pero es seguro que debió haber alguna, puesto que en el mundo helenístico existía una estrecha relación entre la colocación pública de estatuas y sus respectivas inscripciones. Estas eran las encargadas de modificar el significado del monumento, haciendo que el mismo dejara de remitir a personas en particular y capturara la relación entre las personas honradas y las comunidades políticas que las honraban (Ma 2012, p. 241). ¿Qué significaba el Acrocorinto para los aqueos en Argos en algún momento entre la incorporación de la pólis y la llegada de Antígono (229/8-224 a.C.), al menos unos quince años después? La liberación de Macedonia, pero también de los tiranos del Peloponeso, entre ellos, los de Argos (Plut., Arat. 16.2; Plb. II 43.4). Este monumento remitía, pues, al marco social y al escenario político creado durante la acelerada expansión aquea tras la incorporación de Megalópolis (235 a.C.) y de Argos (229/8 a.C.).

2 Cf. Walbank 2006a, pp. 231, 247-248.

${ }^{3}$ IIlion, 25, 11. 116-131.

${ }^{4}$ Liv. XXXI 44.4-8; Flower 2006, pp. 34-41. 
Ambos grupos estatuarios, tanto el de los aqueos como el de Antígono, podrían ser pensados entonces como vectores, o como «vehículos de memoria», que permitían enmarcar estos recuerdos dentro de la experiencia presente para recordar, pero no necesariamente para revivir el hecho (Halbwachs 2004 [1950], pp. 105-138). Arato había mandado levantar las estatuas de los héroes de Acrocorinto no antes del 229/8 a.C., terminus post quem señalado por la incorporación de Argos, pero la toma de esa acrópolis había tenido lugar casi quince años antes. La conmemoración de esta gesta, contra el dominio macedonio en el Peloponeso, tenía un sentido particular para el proyecto político aqueo en Argos. Cuando Antígono mandó restablecer las estatuas de los tiranos argivos, el último de ellos, Aristómaco, había sido ejecutado ese mismo año (224 a.C.), pero el acto de conmemoración de aquella dinastía aliada, sin embargo, tenía importancia para la política antigónida de reingreso en el Peloponeso (Green 1990, p. 261).

El asunto de las estatuas debió resultar, por lo tanto, devastador para la memoria colectiva y también para la identidad de ciertos sectores de la élite política aquea, puesto que la lucha contra la tiranía se había convertido en algo más que una consigna bastante antigua (Orsi 1990, pp. 150-151). Una verdadera identidad política centrada en la democracia como régimen político se había conformado en oposición a la tiranía, aunque esta construcción no alentara necesariamente a la supresión física de los tiranos. Muchos tiranos habían optado, de hecho, por incorporar a sus respectivas póleis a la Confederación y habían recibido a cambio una amnistía por sus delitos pasados. Este no era un camino excluyente, tal como revelan otras experiencias históricas helenísticas.

Muchas póleis helenísticas a partir del s. IV a.C. tuvieron que hacer frente a problemas similares. Por aquella época fueron publicadas varias leyes anti-tiránicas, lo que estaba en relación seguramente con las transformaciones operadas que habían facilitado que la tiranía volviera al centro de la escena política (Mossé 1969, pp. 149-153). La más conocida de estas leyes es la propuesta por Eucrates en Atenas en 337/6 a.C. (SEG XII 87), pero también podría mencionarse una muy completa votada por Ilión en c. 280 a.C. (OGI 218 = Illion, 25) o el llamado expediente de Eresos de c. 306-301 a.C. (OGI 8). Esta última no es estrictamente una ley anti-tiránica, sino una re-publicación de cartas entre Eresos y Alejandro Magno y Antígono Monóftalmos con respecto, en primer lugar, al juicio de los antiguos tiranos de la pólis y, en segundo, sobre una posible readmisión de sus descendientes dentro del cuerpo 
cívico. Como ha señalado recientemente Ellis-Evans (2012), la decisión de publicar estos documentos debe ser entendida como parte de un debate cívico en progreso acerca de cómo interpretar el pasado reciente de la pólis, sobre todo, en relación con el problema político de decretar una amnistía. Eresos decidió no hacerlo y, en consecuencia, se construyó una narrativa histórica acorde para justificar públicamente esta elección. Este caso específico revela que el problema de la tiranía, la amnistía y la política helenística no puede abordarse disociado del de la memoria construida a posteriori en torno al pasado reciente por parte del cuerpo cívico como resultado de luchas y pujas políticas 5 .

En el caso aqueo podemos, por lo tanto, preguntarnos cómo se lograba conciliar aspectos tan definidos de la identidad democrática con la memoria marcada por la experiencia de la tiranía. ¿Hasta qué punto esta negociación entre aqueos y tiranos abría paso efectivamente a un olvido y no se rememoraba activamente la tiranía como contrapunto funcional para la construcción de una identidad colectiva aquea? Conocemos algunos ejemplos de amnistías decretadas por los aqueos. Un primer caso habría sido el de Iseas, tirano de Carinea (c. 276 a.C.) (Plb. II 41.14-15). Posteriormente, la muerte de Demetrio II de Macedonia (229 a.C.) había actuado como un catalizador en el derrumbe de muchos regímenes tiránicos en el Peloponeso, algunos de los cuales se acogieron rápidamente a la generosa oferta aquea. Precedidos por Lidíades de Megalópolis (c. 235 a.C.), Aristómaco de Argos, Zenón de Hermione y Cleónimo de Fliunte renunciaron al poder tras recibir seguridades, regalos y honores (229/8 a.C.) (Plb. II 44.3-6). La oferta de amnistía para quienes renunciaban al poder personal siguió siendo una política sistemática, puesto que durante la campaña contra Nabis (195 a.C.), Aristeno le habría sugerido la posibilidad de obtener una si renunciaba al poder (Liv. XXXIV 33.1-2)

Aunque conocemos esta práctica principalmente a partir de testimonios literarios, existe una posible excepción. El aumento de la presión aquea en el centro del Peloponeso tras el ingreso de Megalópolis empujó a varios tiranos arcadios a seguir el ejemplo de Lidíades, tal como parece sugerir una inscripción de Orcómeno (c. $234 / 3$ a.C. $)^{7}$. Siguiendo el procedimiento habitual

\footnotetext{
5 Ma 2009, p. 256.

${ }^{6}$ Cf. Briscoe 2003, p. 104.

7 Sobre los acontecimientos, v. Walbank 1933, pp. 62-63.
} 
cuando una nueva pólis ingresaba a la Confederación, una homología fundaba la sympoliteía con la Confederación tras regularse primero todas las disputas previas que el nuevo miembro federal poseyera con los antiguos ${ }^{8}$. En el marco de estos arreglos, se menciona una amnistía en favor de un tal Nearco y sus hijos $(I G \mathrm{~V}(2) 344,11.13-17)$. Nada sabemos del caso fuera de este texto epigráfico, pero es difícil suponer que este individuo no fuera el antiguo tirano de Orcómeno. Los aqueos establecían una multa de mil dracmas para todo el que intentara conducir a estos hombres a juicio por delitos cometidos antes de la incorporación de la pólis. El paralelo con lo ocurrido en Argos un par de años más tarde cuando Aristómaco fue absuelto ( $\dot{\alpha} \zeta \eta$ jı $\dot{\varepsilon} \pi \circ i_{\eta} \sigma \alpha \nu$ ) y recibió seguridades ( $\left.\dot{\alpha} \sigma \varphi \alpha \lambda \varepsilon i ́ \alpha \varsigma\right)$ de parte de los aqueos es claro9.

¿Esta cláusula jurídica de amnistía daba paso automáticamente a una voluntad política de olvido? Más bien debería considerarse esta práctica como una estrategia de «olvido recordado», que no buscaba abrir paso a una amne$\operatorname{sia}^{10}$. El juramento solemne realizado por los magistrados aqueos y los orcomenios, así como la colocación de una copia del documento en el centro político federal en Egio, tenían como objetivo proporcionar seguridad jurídica al ex-tirano. Este obtenía garantías de no ser perseguido, pero con ello paradójicamente se evitaba el olvido, puesto que la inscripción actuaba como una marca en el espacio público y brindaba un soporte para el trabajo subjetivo y la acción colectiva (Jelin y Langland 2003, p. 4). Su presencia recordaba que, por alguna razón, se había perdonado, puesto que la principal motivación para la colocación de copias públicas de documentos de una pólis era su contribución a la memoria colectiva cívica (Carlsson 2010, p. 157).

En ese sentido, puede entenderse también la reacción de Arato por el asunto de las estatuas de los tiranos argivos. Su sustitución por las de los héroes aqueos, que habían liberado el Acrocorinto, constituía una operación de memoria sobre el espacio público de la pólis. Este monumento ayudaba a los argivos a recordar su nuevo vínculo con el pasado aqueo inmediato, de guerra contra Macedonia y contra las tiranías, que ahora, desde su incorporación se había convertido en su propio pasado. Este monumento recordaba a los argivos el doble objetivo federal de Arato: la expulsión de los macedo-

${ }^{8}$ Cf. Epidauro (Ager 1996, n.38); Koehn 2013, p. 177.

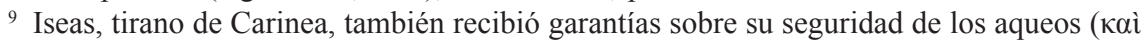

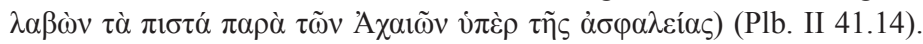

${ }^{10}$ La falsa equivalencia amnistía/amnesia para el caso ateniense: Loraux 2008, pp. 145-169. 
nios y la abolición de las tiranías ${ }^{11}$. Situar estas estatuas donde la ciudadanía estaba acostumbrada a observar aquellas de los tiranos permitía ligar ambos grupos estatuarios, convirtiéndose a la vez en un ejercicio y en una convocación abusiva de la memoria ${ }^{12}$.

Lidíades de Megalópolis, ex-tirano y político relevante dentro de la Confederación, que fue tres veces estratega federal, había experimentado este choque con el recuerdo activo de la tiranía. Plutarco señala que el motivo por el cual él nunca había obtenido del todo la confianza de los aqueos, a diferencia de Arato, era que había sido un tirano (Plu., Arat. 30.5). En el marco de las negociaciones para la entrada de Argos en la Confederación Aquea, Lidíades recomendaba a Aristómaco que no se fiara de Arato, ya que este era reconocido por su odio visceral contra los tiranos ${ }^{13}$. El haber sido tirano se convertía, a pesar de la amnistía, en un estigma que marcaba la carrera política y, en la práctica, era una muestra de la existencia de una verdadera memoria de la tiranía dentro de la élite política aquea.

\section{LA EJECUCIÓN DE ARISTÓMACO Y LOS LÍMITES DEL OLVIDO DEL RÉGIMEN TIRÁNICO}

En 224 a.C. se produjo una insurrección en Argos, que había entrado en la órbita de Cleómenes III el año anterior y se había separado de la Confederación aquea. Este movimiento fue rápidamente apoyado desde afuera por el estratega aqueo Timóxeno, quien avanzó desde Sición y reconquistó la pólis. La acción fue decisiva para forzar al rey espartano a abandonar su posición estratégica en el istmo de Corinto y facilitar así el cruce de Antígono con sus fuerzas al Peloponeso. Las circunstancias de la previa defección de Argos en 225 a.C. han quedado, sin embargo, en la nebulosa, puesto que los testimonios históricos son bastante escasos y contradictorios.

${ }_{11}$ Plb. II 42.3; 43.8. La toma de Acrocorinto es comparada por Plutarco con la empresa del tebano Pelópidas y la del ateniense Trasibulo, aunque superior pues fue contra un extran-

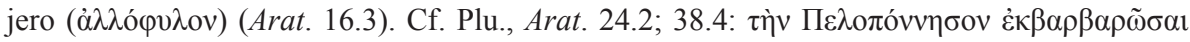

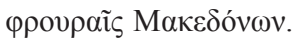

12 «acordarse es no sólo acoger, recibir una imagen del pasado; es también buscarla, 'hacer' algo... El verbo designa el hecho de que la memoria es 'ejercida'» (Ricoeur 2004, p. 81).

${ }_{13}$ Plu., Arat. 35.1. Cf. Paus. II 8.6. El odio de Arato por los tiranos (Plu., Arat. 3.1; 10.1; $26.3 ; 28.4)$. 
Particularmente enigmáticos resultan los sucesos relacionados con la captura y ejecución de Aristómaco. La carrera de este individuo es relativamente bien conocida (Wilcken 1895). Hijo de Aristómaco I y hermano de Aristipo II, sucedió a este en el poder tiránico como Aristómaco II cuando aquel cayó en una emboscada de las fuerzas de Arato cerca de Cleonas (235 a.C.) (Plu., Arat. 27-29.5). Con la ayuda de tropas de Demetrio II pudo asumir el poder, que detentó hasta 229/8 a.C. en que se decidió a abandonar la tiranía y unir a Argos a la Confederación, que lo eligió inmediatamente estratega (228/7 a.C.) (Plb. II 44.6; Plu., Arat. 35.3; cf. Paus., II 8.6). Los hechos ocurridos durante la Guerra Cleómenica son confusos, puesto que, dependiendo de la fuente, Argos se habría separado o habría sido tomada a traición por Cleómenes (225 a.C.) (Plu., Cleom. 17.4-5; Arat. 39.5)14. Para el 224 a.C. los aqueos habían retomado el control y Aristómaco había sido ejecutado en Céncreas (Plb. II 59.1, 60.7, 61.8; Plu., Arat. 44.4).

Estos sucesos son expuestos por el historiador aqueo Polibio de Megalópolis, quien fue además un destacado miembro de la élite política federal. En un polémico pasaje contra Filarco, aquel justificaba esta ejecución y, al hacerlo, revela, en nuestra opinión, los límites del olvido de su amnistía (Plb. II 59-60). En efecto, su retórica anti-tiránica alcanza en aquella oportunidad una notable virulencia, puesto que señala que, como Aristómaco era un tirano, sólo por ese motivo merecía el peor de los castigos, añadiendo a las acusaciones su traición a la Confederación, que le había dado previamente

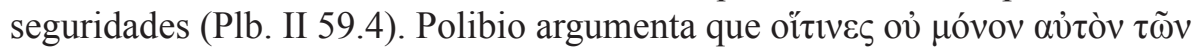

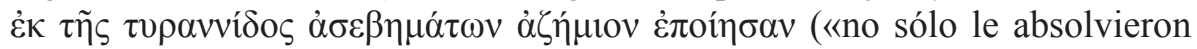
de los crímenes de la tiranía, sino que le recibieron en la Confederación y le confiaron la máxima dignidad») (Plb. II 60.5). ¿Por qué recurrir al argumento del pasado tiránico para justificar su ejecución? ¿La amnistía no borraba ese pasado? ¿No bastaba acaso con aludir a su traición a los aqueos?

Lo primero que habría que aclarar es hasta qué punto la justificación de Polibio puede ser considerada una reproducción de las excusas por la muerte de Aristómaco expuestas originalmente en las Memorias de Arato. Es sabido que el historiador aqueo utilizó esta «autobiografía» como fuente para los acontecimientos en Grecia hasta el 221 a.C. (Walbank 1999a, p. 228; Pédech

14 Ambos pasajes revelan probablemente fuentes diversas utilizadas por Plutarco, siendo estas Filarco y Arato respectivamente (Ferrabino 1972, pp. 96, 302; Cf. Marasco 1981, pp. 490-491). 
1964, p. 261), 220 a.C. (Errington 1967, p. 20, n.9) o, tal vez, el 219 a.C., si se contempla la utilización adicional de unos posibles informes diarios (ephemérides) sobre la campaña en Tesalia y Grecia central (Plb. V 97-101) (Meadows 2013). Se conoce también que estas Memorias tenían un marcado tinte apologético, que buscaba justificar la acción política de su autor (Engels 1993, pp. 32-33). Sin embargo, también debe tenerse en cuenta que cualquier estudio de Quellenforschung es especulativo, puesto que dichas Memorias no se han conservado.

Sería más útil, por el contrario, entender esta acusación de Polibio no como una construcción puramente original, ni tampoco como una reproducción textual de las acusaciones formuladas por Arato. Pudo ser más bien una mezcla de ambas. Las coincidencias podrían entenderse como una expresión de solidaridad con la posición política de Arato, pero, también, y fundamentalmente, como el resultado de una visión política compartida por dos miembros de la élite política aquea ${ }^{15}$. Desde esta perspectiva, el concepto de «habitus» de Bourdieu (2010 [1980], pp. 85-92), entendido como el producto de la historia personal, pero también del espacio social en el cual se desarrollan las prácticas, que se conforma como una verdadera disposición duradera para pensar, actuar y hablar de una determinada manera por parte del agente social, puede ayudar a comprender que la producción libre de los pensamientos, percepciones y acciones de este se da siempre inscrita dentro de condiciones histórica y socialmente situadas. La Confederación Aquea era una democracia, lo que Polibio celebraba y compartía en consonancia con las inscripciones aqueas $\left(S I G^{3}\right.$ 665.17-19). Una visión compartida del pasado aqueo se había conformado en la élite política, que entendía este como una experiencia de lucha constante contra las tiranías peloponesias. Polibio compartía con Arato este «habitus», esta disposición para ver y actuar. No es sorprendente así que la oposición entre democracia y tiranía aparezca en su obra en contextos que no dependen necesariamente de Arato como fuente, tales como la muerte de Pelópidas a manos del tirano Alejandro, lo que revela una interiorización de esta lectura política del pasado (Plb. VIII 35.6). El concepto de «habitus» puede hasta cierto punto, además, articularse con el de memoria colectiva, en tanto que el primero se refiere a la «historia hecha cuerpo» por los agentes sociales y el segundo, según Halbwachs, a las representaciones

15 Sigue a Arato como fuente como una opción política, no historiográfica (Klatt 1877, p. 26). 
del pasado compartidas por un determinado grupo. Es decir, la memoria colectiva contribuye a la conformación del «habitus», pero este último también colabora en la constante y dinámica redefinición de la memoria. Ahora bien, la memoria, aunque es un fenómeno social colectivo, sólo es reconocible a partir de las acciones y los recuerdos de individuos concretos (Kansteiner 2002, pp. 185-190). ¿No podríamos ver, pues, en Polibio un ejemplo de recepción de esta memoria aquea de la tiranía? Una mirada a la Achaica (II.37-70) confirma esta lectura:

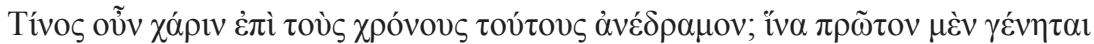

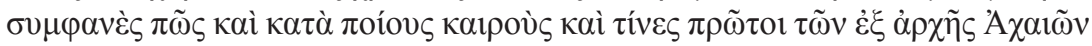

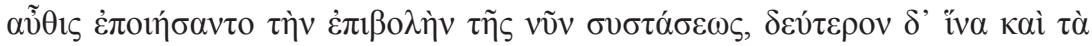

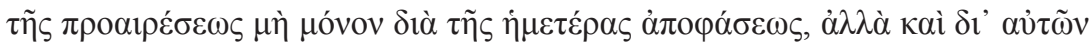

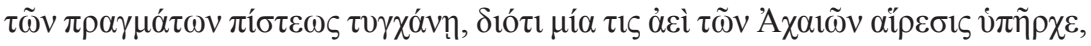

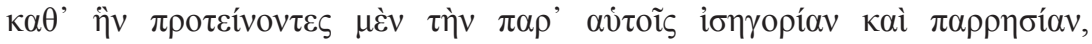

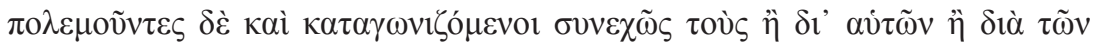

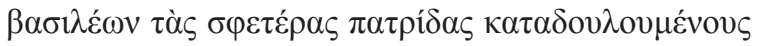

¿En razón de qué, pues, me he remontado a estos tiempos? En primer lugar, para que resulte claro cómo y en qué época y quiénes fueron los primeros, de entre los antiguos aqueos, que llevaron a cabo el proyecto de la actual federación. Y, en segundo lugar, para que lo relativo a la política adquiera, no por mi afirmación, sino por los hechos mismos, la credibilidad de que el ideal político de los aqueos ha tenido siempre una cierta unidad, de suerte que tomándolo como modelo, ofrecieron a los hombres la igualdad y la libertad de expresión de que ellos gozaban al tiempo que hacían la guerra y desafiaban, sin tregua, a cuantos, ya por sus propias fuerzas, ya con la ayuda de los reyes, intentaban esclavizar a sus respectivas patrias (Plb. II 42.1-3, trad. Díaz Tejera 1986).

El texto muestra hasta qué punto se integraba dentro de la representación aquea del pasado, hecha cuerpo por Polibio, un «relato», una particular lectura del pasado, de oposición histórica entre liberación y democracia, por un lado, y tiranía y esclavitud, por el otro. Por su «habitus», conformado dentro del campo político de la élite aquea, el historiador percibía la interpretación de Arato no sólo como razonable, sino como la única pensable. En su discurso aparecen indicios de esta representación del pasado aqueo construida oposicionalmente, como se observa explícitamente en su oposición entre tiranía y democracia en el relato de la batalla de Mantinea contra el «tirano» 
Macánidas (Plb. XI 13.4-8). En realidad, no parece que Macánidas haya sido «tirano», sino en realidad regente del hijo del rey Licurgo, Pélope ${ }^{16}$. En esta batalla Filopemén buscó deliberadamente matar con sus propias manos al «tirano» (XI 18.4, 17.2). No era la primera vez que un tirano resultaba muerto en combate contra los aqueos, puesto que esto le había ocurrido a Aristipo de Argos (Plu., Arat. 29.3-4). Si creemos a Plutarco, la posición en la que Filopemén dio muerte al «tirano» habría sido la misma en la que luego fue representado con una estatua dedicada por los aqueos en Delfos ${ }^{17}$. Matar a un tirano era, pues, el mayor honor para un ciudadano aqueo y se buscó exhibirlo públicamente como un buen ciudadano, es decir, como un matador de tiranos. El mensaje ético era claro: «a los que eliminan y castigan a un tirano los hombres sensatos les rinden aprobación y honores» (Plb. II 60.2) ${ }^{18}$.

Este razonamiento, con todo, ha sido tachado de ilógico, pues, tras incorporarse Argos a la Confederación, Aristómaco había recibido una amnistía oficial y sus crímenes pasados como tirano habían sido, por lo tanto, perdonados. De hecho, no existen pruebas y, de tenerlas a mano, Polibio seguramente las hubiera aportado, de que aquel individuo hubiera reasumido la tiranía durante la breve secesión de Argos (Walbank 1999a, p. 265). El historiador aqueo sólo menciona un ataque sorpresa por parte de Arato y, tras su fracaso, la ejecución de 80 cómplices internos, pero estos hechos se remontaban al año 235 a.C., cuando Aristómaco recién se había hecho con el poder y, por lo tanto, antes de recibir una amnistía (Plb. II 59.7-9; Walbank 1999a, p. 266). ¿Cuál fue la participación de Aristómaco en la secesión argiva? Plutarco sólo menciona que la pólis se había pasado a Cleómenes, pero no dice nada sobre el ex-tirano (Plu., Arat. 39.4) ${ }^{19}$. Hubiera sido coherente mencionarlo en ese contexto, precisamente, porque los movimientos en favor de Cleómenes se atribuían a las ambiciones personales de algunos líderes (Plu., Arat. 40.1; Orsi 1991, p. 24). Sin embargo, no hay ninguna mención a una vuelta al poder. Por otra parte, la presentación de Filarco no sugiere que fuera tirano en el momento de su ejecución, sino que lo había sido en el pasado

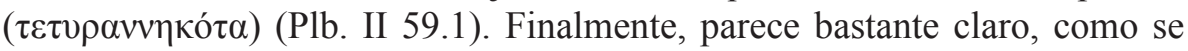

16 Liv., XXVII 29.9. Macánidas es tyrannus Lacedaemoniorum, «tirano de los lacedemonios», aunque Pélope estaba todavía vivo (D. S. XXVII 1); Errington 1969, p. 55, n. 1.

${ }_{17}$ Plu., Phil. 10.7-8; Vatin 1975, pp. 211-217.

${ }_{18}$ Pudo hacerse eco de la retórica anti-tiránica común en algunas leyes griegas (Teegarden 2007).

${ }^{19}$ Cf. Plu., Cleom. 17.5. 
desprende del episodio de la rebelión de Aristóteles del 224 a.C., que Argos estaba directamente bajo el control de Cleómenes (Plu., Arat. 44.2). Puede dudarse, por lo tanto, de la pertinencia del juicio polibiano, no sólo porque el argumento «tiránico» debía haber desaparecido tras la amnistía, sino también porque no parece que Aristómaco hubiera reasumido el poder en Argos entre 225 y 224 a.C.

Si este no volvió a ser tirano, como es la conclusión más lógica, ¿por qué Polibio consideró mejor centrarse en este argumento? Naturalmente, él estaba contestando a Filarco, quien había aludido al ilustre origen de este hombre como tirano y descendiente de tiranos de Argos (Plb. II 59.1). Según el mismo Polibio, Aristómaco había sido amnistiado por los aqueos. Pese a esto, prefería poner el acento en que «aunque no hubiera cometido delito alguno contra los aqueos, por su talante político durante su vida y por su traición a su patria, se hacía digno del más acerbo castigo» ${ }^{20}$. La traición original era haber detentado la tiranía, lo que llevaba a una totalización de su carácter

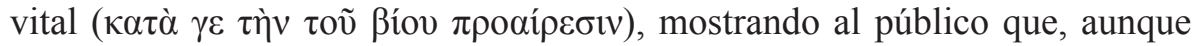
hubiera renunciado y hubiera sido amnistiado, en esencia, nunca había dejado de ser un tirano. Era una acción perfectamente coherente con la memoria aquea, que Polibio compartía no sólo en la Achaica sino a lo largo de toda su obra.

La defensa por el proceder de Antígono y los aqueos, que habían sido acusados de crueldad por Filarco, resulta además extralimitada sobre la base de una reconstrucción absolutamente arbitraria de los acontecimientos (Plb. II 59.1). Se advierte en la misma un solapamiento frecuente entre memoria e historia, entendiendo que ambas son selecciones, interpretaciones y distorsiones inconscientes o conscientes y siempre socialmente condicionadas (Burke 1989, p. 98). Es más factible pensar la ejecución de Aristómaco como la decisión de Antígono, puesto que este tenía cuentas pendientes con el antiguo tirano, quien le había traicionado en el pasado para pasarse a los aqueos. Es más, el temor a sus represalias es lo que pudo volcar a Aristómaco y a sus seguidores del lado de Cleómenes para evitar un peligroso acuerdo con Macedonia (Walbank 2006b, p. 466). En ese sentido, no puede dejar de advertirse que la ejecución fue en Céncreas, uno de los dos puertos corintios, y se sabe que esta pólis había pasado a dominio macedonio inmediatamente des-

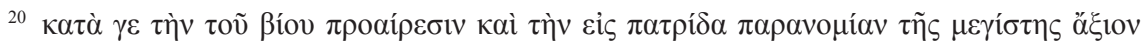

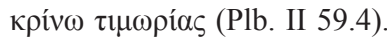


pués de la retirada de Cleómenes ${ }^{21}$. Por lo demás, esto coincidiría con la noticia de una masacre perpetrada en Argos por el macedonio Leoncio tras la partida de Antígono, en la que se habría eliminado quizá a los responsables del acuerdo con Cleómenes (Plb. V 16.6). Si fue ejecutado por los macedonios, ¿por qué Polibio, entonces, escribió que lo más justo hubiera sido hacer con este un castigo ejemplar tras conducirlo por el Peloponeso? (Plb. II 60.7). La respuesta es que el castigo debió ser público, no nocturno y en una casa como había sugerido Filarco (Plb. II 59.2), pero también que debió haber sido aplicado por los propios aqueos porque matar a un tirano era el máximo honor cívico. Existe aquí también poco olvido de la tiranía.

Por lo tanto, existen indicios de un recuerdo de la tiranía ejercido entre los aqueos. Así, en Argos, aunque se había amnistiado a un tirano, sus estatuas y las de su familia habían sido eliminadas y se había colocado en su lugar un monumento como marca de la gesta liberadora de los héroes del Acrocorinto. En Orcómeno, aunque se había establecido un castigo a todo aquel que pretendiera llevar ante los tribunales a un ex-tirano o a sus hijos, se había colocado la inscripción en un lugar público del centro político aqueo en Egio, volviendo la amnistía una marca también de no-olvido. Finalmente, en el caso de Polibio, y su justificación de la ejecución de un tirano, la marca de la lucha contra los tiranos se hallaba hecha cuerpo por un historiador que era, a su vez, miembro de la élite política aquea, y ello revelaba nuevamente los límites de la amnistía.

IV. El motivo ANTI-TIRÁNiCo EN EL S. II A.C. Y LOS Límites DE LA MEMORIA EJERCIDA

¿Qué consecuencias tenía este no-olvido de los regímenes tiránicos? Como muestra Koehn (2007, pp. 135-155), el discurso anti-tiránico llegó a convertirse en una eficaz herramienta de legitimación del expansionismo aqueo. No se trataba de un discurso que persiguiera necesariamente la supresión física de los tiranos, sino la desaparición de sus regímenes y, de hecho, la negociación y la política de amnistías constituían un camino político preferible.

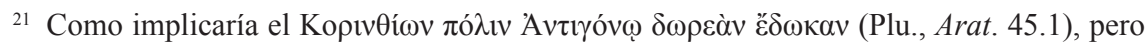
también la noticia de que, a la huída de Cleómenes de Corinto, este perdió la ciudad porque Antígono entró en ella y colocó una guarnición (Plu., Cleom. 21.3). Nuestra reconstrucción difiere de la de Tomlinson 1972, pp. 160-161. 
Esta voluntad política de negociación no anulaba, de todos modos, el fenómeno paralelo de activa construcción del pasado reciente aqueo asimilado a una guerra de liberación del Peloponeso de manos de los tiranos enemigos. Anuladas las tiranías en el área norte y centro de esta península, se abrió una segunda etapa de expansión aquea contra Esparta, cuyos reyes fueron víctimas de la misma retórica.

Se ha señalado ya que Aristeno había ofrecido a Nabis de Esparta la posibilidad de renunciar a la tiranía y de beneficiarse, por lo tanto, de una amnistía aquea. El intento finalmente no fue exitoso y Nabis cayó asesinado por sus propios aliados etolios (192 a.C.), lo que permitió a Filopemén aprovechar el vacío consiguiente de poder en el sur para apoderarse de Esparta, que a partir de allí se convirtió en un miembro peligroso para la estabilidad interna de la Confederación. En 191 a.C., a un año de su incorporación, se produjo un primer intento de secesión y, en 188 a.C., un segundo, lo que recién se alcanzaría en 146 a.C. con la intervención de Roma. Se conservan dos epigramas que celebraban la supresión del segundo movimiento secesionista por Filopemen ${ }^{22}$. Pausanias habla también sobre una inscripción honorífica en la base de una estatua de este líder aqueo en Tegea:

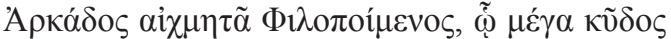

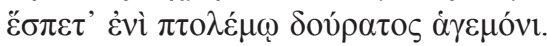

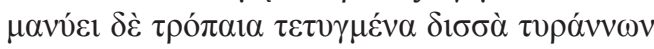

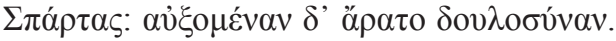

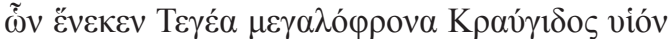

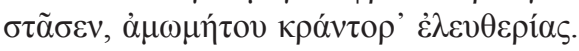

Un guerrero arcadio, Filopemen, al que una gran gloria acompañó como jefe de las lanzas en la guerra, lo indican dos trofeos tomados de los tiranos de Esparta. Destruyó la esclavitud creciente, a causa de lo cual Tegea, al hijo magnánimo de Craugis, lo erigió, jefe de libertad irreprochable (Paus., VIII 52.6; trad. Herrero Ingelmo 1993).

Esta inscripción estaría en relación también con la liberación de la ciudad por Filopemen, tras su victoria en Mantinea sobre Macánidas (207 a.C.), que

${ }^{22}$ POxy. 662 col. II. 32-40 (= Page 1942, pp. 455-456); AP VII 723 (= Rizakis 1995, p. 72). 
murió a manos de aquel (Plb. XI 18.7-8). Se advierten así huellas del «habitus» construido de acuerdo con la memoria colectiva de la élite política aquea, de su disposición para pensar y actuar, interpretando el presente como el resultado de una lucha histórica entre tiranía y esclavitud, por un lado, y democracia y libertad, por el otro. Dentro de esta orgullosa celebración colectiva de victoria contra el enemigo tiránico, se entiende una noticia sobre la entrada de Filopemen en el teatro acompañado de sus jóvenes soldados durante la celebración de los Juegos Nemeos (205 a.C.). Allí comenzaron a recitarse Persas de Timoteo que versaba sobre el tema de la ilustre libertad y el orden obtenidos para Hélade tras la derrota de los bárbaros orientales (Plu., Phil. 11.2). La asociación entre el gran tirano persa y Macánidas era evidente.

El mismo patrón ideológico se advierte en el lenguaje político de Polibio. Por ejemplo, Nabis parece haber sido rey de Esparta, título que adoptaba en inscripciones y monedas, pero en las Historias, por el contrario, se convirtió en el prototipo del tirano enemigo de la libertad (Walbank 1999b, pp. 419420; cf. Texier 1975, p. 16) ${ }^{23}$. Es muy probable que la imagen de los reyes espartanos se moldeara sobre la experiencia de Cleómenes, el primer gran «tirano» al que los aqueos enfrentaron. Esto podría considerarse como una huella, que, como la entiende Ginzburg 2010, permite seguir el hilo del engaño hasta su unión con la verdad o, en términos de memoria e historia, el indicio que nos puede permitir desmontar esta construcción cultural aquea definida a partir de su experiencia presente de lucha por la hegemonía sobre el Peloponeso. Walbank (1999b, p. 420) señala que la tradición de Nabis como tirano se remonta efectivamente a Polibio, pero esto es inexacto. La inscripción que Pausanias halló en Tegea parece mostrar que ya en época de Filopemen existía una asociación de los líderes espartanos con la tiranía, así como una construcción de los aqueos como liberadores.

El fantasma de la tiranía como «otro» de la democracia aquea no desapareció cuando Esparta fue incorporada. La violencia aquea contra los espartanos tendió a intensificarse. En ese contexto, el recuerdo de la tiranía continuó operando como un esquema de percepción y justificación, fundamentalmente, a partir de la insistente presión de los espartanos exiliados por Nabis. En un primer momento, cuando Esparta fue incorporada, no se permitió su reincorporación debido a los problemas que esto podía ocasionar durante la

\footnotetext{
${ }^{23} I G \mathrm{~V}(1) 885 ; S I G^{3} 584$, etc.
} 
guerra contra Antíoco III. La situación cambió pronto cuando se produjo el segundo intento secesionista ${ }^{24}$. Tras la masacre de Compasio y la ejecución de 80 líderes espartanos, según Polibio, y 350 según Aristócrates, se tomó una serie de medidas adicionales contra Esparta ${ }^{25}$. Fueron derribados sus muros; se expulsó a quienes habían militado como mercenarios bajo los tiranos; se desterró a los ilotas liberados, dándoles un plazo para abandonar el territorio espartano; fueron abolidas las leyes de Licurgo e impuestas las aqueas y, finalmente, se decretó el retorno de los exiliados (Liv. XXXVIII 34.1-4; Plu., Phil. 16.3-4; cf. Paus. VIII 51.3).

Se trataba de un paquete de medidas que buscaba quebrar las tendencias separatistas espartanas, incorporándola plenamente a la sympoliteía aquea y debilitando al mismo tiempo los apoyos internos del antiguo régimen «tiránico». La expulsión de los antiguos mercenarios y de los ilotas previamente liberados se asociaba a este intento. Polibio explica que, cuando la batalla de Mantinea estaba ya decidida, Filopemen había ordenado no perdonar la vida de ningún mercenario, pues estos habían sido siempre en el pasado los sostenedores de las tiranías (Plb. XI 18.1). Tras las medidas del 188 a.C., muchos de los antiguos mercenarios e ilotas habían cumplido la orden de abandonar la ciudad, pero se habían dispersado por la campiña (Liv. XXXVIII 34.6). La respuesta de la asamblea aquea fue enviar a Filopemén con tropas a tomar a estos hombres como botín. Tito Livio omite la cifra de estos, pero Plutarco menciona un total de 300 (Plu., Phil. 16.4).

Con el dinero procedente de su venta, se tomó la decisión de reconstruir un pórtico en Megalópolis (Liv. XXXVIII 34.7; Plu., Phil. 16.4). Errington $(1969$, p. 147) ha sugerido que esto fue un vano gasto en un «victory display» de valiosos recursos que podrían haber servido para normalizar la incorporación de la pólis ${ }^{26}$. En su momento, Arato había conseguido los 50 talentos necesarios para pagar las soldadas adeudadas a los mercenarios de Aristóma-

${ }^{24}$ Datación de los acontecimientos entre 189/188 a.C.: Briscoe 2008, p. 115.

25 Plutarco (Phil. 16.4) señala que 80 es la cifra de Polibio y 350 la de Aristócrates $(F G r H$, 591 F4). Livio (XXXVIII 33.10-11) parece aceptar la cifra de Polibio (septemdecim... sexaginta tres). Los 350 puede ser una exageración pro-espartana, producto quizá de represalias posteriores (Errington 1969, p. 145). Pausanias (VIII 51.3) sólo señala que 300 individuos fueron expulsados del Peloponeso.

${ }^{26}$ Seguido por Walbank 1999c, p. 139; Briscoe 2008, p. 117. Golan 1974 ha concluido crudamente que las acciones de Filopemén estaban dirigidas a oprimir a otros griegos más que a oponerse a los romanos. 
co en Argos, eliminando una potencial causa de conflicto, y, en este caso, el dinero proveniente de esos esclavos podría haber contribuido a zanjar diferencias económicas entre los ciudadanos espartanos exiliados y los que habían permanecido, sobre todo, con respecto al crucial problema de la tierra ${ }^{27}$. Un pasaje de Polibio, procedente del De Sententiis, sin embargo, presenta las decisiones tomadas en aquella oportunidad por Filopemen como bellas y convenientes (Plb. XXI 32c). Bello ( $\alpha \alpha \lambda o ́ v)$ fue «restituir a la patria a los

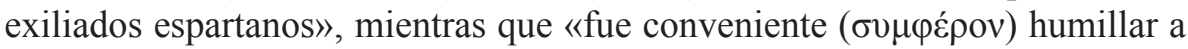
la ciudad de los espartanos, desterrando a los que habían servido a la dinastía de los tiranos ${ }^{28}$. La tiranía no moría con el individuo que la detentaba. Desaparecido Nabis en 192, no sólo Diófanes en 191, sino también Filopemén en 188 a.C. habían tenido que lidiar con su fantasma, que descansaba, en última instancia, en una desigual estructura socio-económica. Los conflictos limítrofes, como el de 163 a.C. entre Megalópolis y Esparta, contribuyeron a profundizar las tendencias centrífugas dentro del edificio federal. Es significativo que la mediación entre estas dos ciudades sea el único documento oficial aqueo donde se explicita el carácter democrático de la Confederación $\left(S I G^{3} 665,11.17-19\right)$, quizá como una forma de conjurar una amenaza.

De acuerdo con el tenor de la inscripción hallada en Tegea por Pausanias, la reconstrucción del pórtico en Megalópolis podía tener un significado especial ligado a la memoria aquea de lucha contra la tiranía. Es muy probable que el pórtico reconstruido con el dinero proveniente de los despojos de la última «tiranía» fuera el mismo que se menciona como erigido originalmente para celebrar la victoria sobre Acrótato y los espartanos ${ }^{29}$. Este debió haber sido destruido cuando Cleómenes tomó y saqueó Megalópolis (223 a.C.) (Plb. II 55.2-7). Al usar los recursos provenientes de los restos del último régimen «tiránico» espartano para reparar los daños cometidos por el primero contra el cual los aqueos se habían enfrentado, se estaba haciendo un ejercicio activo de memoria y conmemoración. El «victory display» consti14).

${ }^{27}$ Algo similar había enfrentado Arato en Sición tras liberarla de los tiranos (Plu., Arat.,

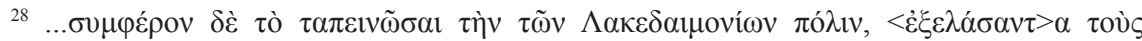

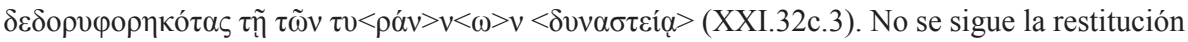

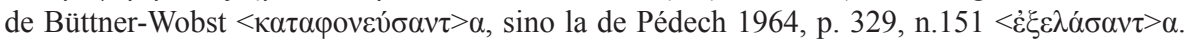

${ }^{29}$ Acrótato era el hijo de Areo, derrotado posiblemente c. 255 a.C. (Walbank 2006a, p. 231). El pórtico descripto (Paus. VIII 30.7; Cf. Plu., Agis 3.7; Paus. VIII 27.11). Ver: Urban 1979, pp. 43-44. 
tuía una respuesta, monumentalizada, a las afrentas históricas de los tiranos espartanos perfectamente coherente con una lectura maniquea del pasado, que no reparaba en el hecho de que los espartanos eran ahora también aqueos, tanto como los megalopolitanos. Estos monumentos estaban marcados, por lo tanto, por las heridas históricas abiertas dentro de la Confederación, y reforzaban las rivalidades entre póleis, promoviendo una política sistemática de no-olvido. Ese pórtico se erigía ahora en Megalópolis para volverse una marca material, visible, de la victoria de unos y la humillación de otros. Como señala Assmann, no es tan fácil decretar el olvido como apagar un micrófono, pero una solución que hubiera permitido una incorporación menos traumática de Esparta hubiera sido la negociación de un pasado común para «destruir el estrecho horizonte de la propia memoria colectiva» (Assmann 2008 , p. 41$)^{30}$.

\section{Conclusión}

La incapacidad para negociar una incorporación equitativa, pero también para redefinir una memoria común, que hubiera requerido re-semantizar la experiencia de los regímenes tiránicos, condujo, junto con otras contradicciones de tipo socio-económico, finalmente al alzamiento de Esparta y a la disolución de la Confederación por los romanos en 146 a.C. Antes de que ello ocurriera, vencida Esparta e incorporada, los aqueos habían podido celebrar efímeramente la unidad del Peloponeso bajo una identidad a la vez democrática y anti-tiránica. Así lo hicieron, entregándose a un "victory display». Esa unidad, sin embargo, lograda sobre la humillación del «otro» y sobre la base de una política de memoria selectiva, que no dejaba un lugar para el pasado de Esparta dentro de esa historia que se pretendía común, resultó ser una bomba de relojería para el edificio federal.

Del mismo modo, la supuesta, pero no probada, rebelión de Aristómaco, ocurrida en el marco de la lucha entre la Confederación Aquea y Cleómenes de Esparta por la hegemonía sobre el Peloponeso, no buscaba ser racionalizada por Polibio como la consecuencia lógica de un cambio en las relaciones de poder al interior del cuerpo ciudadano argivo y de la agudización de la

${ }^{30}$ La política de conciliación de Calícrates pudo haber tenido ese objetivo (Didu 1993, p. 31, n. 69). El agradecimiento de los exiliados espartanos a quienes había facilitado su regreso en el 179/8 a.C. $\left(S I G^{3} 634\right)$. 
stásis, sino simplemente como la acción de un ambicioso individuo que buscaba retomar la tiranía. El enemigo, interno o externo, era siempre identificable, puesto que la historia aquea era percibida como una lucha constante contra los reyes de Macedonia y sus ambiciosos aliados tiránicos. Se evitaban de ese modo comprometedoras preguntas acerca de la solidez del orden político establecido por los aqueos, sobre el grado de consenso y aceptación del mismo por parte de amplios sectores del cuerpo cívico de las póleis peloponesias, y se reducía el movimiento separatista a la traición de un individuo. Pese a haber sido amnistiado y perdonado, se pretendía demostrar discursivamente que Aristómaco jamás había dejado de ser un tirano enemigo de los aqueos, es decir, una amenaza para el nuevo orden peloponesio. La generosa política de amnistías ofrecidas por la Confederación Aquea a aquellos tiranos que abandonaran el poder otorgaba seguridad jurídica a estos individuos, pero no traía aparejada una política de olvido, sino un ejercicio y construcción de la memoria como política sistemática de reforzamiento de la identidad de la élite política federal.

\section{BIBLIOGRAFÍA}

Ager, S. 1996: Interstate Arbitrations in the Greek World (337-90 B.C.), Berkeley. Assman, J. 2008: Religión y memoria cultural. Diez Estudios, Buenos Aires.

Aymard, A. 1938: Les Assemblées de la Confédération Achaienne. Étude Critique d'Institutions et d'Histoire, Burdeos.

Aymard, A. 1970 [1938]: Les premiers rapports de Rome et de la Confédération Achaienne (198-189 avant. J.-C.), Roma.

Bastini, A. 1987: Der achäische Bund als hellenische Mittelmacht. Geschichte des achäischen Koinon in der Symmachie mit Rom, Fráncfort del Meno.

Beck, H. 1997: Polis und Koinon. Untersuchungen zur Geschichte und Struktur der griechischen Bundesstaaten im 4. Jahrhundert v. Chr., Stuttgart.

Bourdieu, P. 2010 [1980]: El Sentido práctico, Buenos Aires.

Briscoe, J. 2003 [1981]: A Commentary on Livy. Books XXXIV-XXXVII, Oxford.

Briscoe, J. 2008: A Commentary on Livy. Books XXXVIII-XL, Oxford.

Burke, P. 1989: «History as Social Memory», en Butler, Th. (ed.), History, Culture, and the Mind, Nueva York, pp. 97-113.

Carlsson, S. 2010: Hellenistic Democracies. Freedom, Independence and Political Procedure in Some East Greek City-States, Stuttgart.

Didu, I. 1993: La fine della Confederazione Achea. Lotta politica e rapporti con Roma dal 180 al 146 a.C., Cagliari. 
Dubois, M. 1885: Les ligues étolienne et achéenne. Leur histoire et leurs institutions. Nature et durée de leur antagonisme, París.

Ellis-Evans, A. 2012: «The Tyrants Dossier from Eresos», Chiron 42, pp. 183-212. Engels, J. 1993: «Die 'YПOMNHMATA-Schriften und die Anfänge der politischen Biographie und Autobiographie in der Griechischen Literatur», ZPE 96, pp. 19-36.

Errington, R. 1967: «Philip, Aratos and the 'Conspiracy of Apelles'», Historia 16, pp. 19-36.

Errington, R. 1969: Philopoemen, Oxford.

Ferrabino, A. 1972 [1921]: Il problema dell'unitá nazionale nella Grecia antica, vol. 1, Arato di Sicione e l'idea nazionale, Roma.

Flower, H. 2006: The Art of Forgetting. Disgrace and Oblivion in Roman Political Culture, Chapel Hill.

Freeman, E. 1863: History of Federal Government from the Foundation of the Achaian League to the Disruption of the United States, vol. 1, Londres-Cambridge.

Gangloff, A. (ed.) 2013: Lieux de mémoire en Orient grec à l'époque impériale, Berna.

Ginzburg, C. 2010 [2006]: El hilo y las huellas. Lo verdadero, lo falso, lo ficticio, Buenos Aires.

Golan, D. 1973: «Aratus' Policy between Sicyon and Argos: An Attempt at Greek Unity», RSA 3, pp. 59-70.

Golan, D. 1974: «Philopoemen immodicus and superbus and Sparta», SCI 1, 1974, pp. 29-39.

Grainger, J. 1999: The League of the Aetolians, Leiden.

Green, P. 1990: Alexander to Actium. The Historical Evolution of Hellenistic Age, Berkeley.

Haake, M. y Jung, M. (eds.) 2011: Griechische Heiligtümer als Erinnerungsorte: von der Archaik bis in den Hellenismus, Stuttgart.

Halbwachs, M. 2004 [1950]: Los marcos sociales de la memoria, Barcelona.

Jelin, E. y Langland, V. 2003 [2002]: «Introducción: Las marcas territoriales como nexo entre pasado y presente», en Jelin, E. y Langland, V. (comps.), Monumentos, memoriales y marcas territoriales, Madrid, pp. 1-18.

Kansteiner, W. 2002: «Finding Meaning in Memory: A Methodological Critique of Collective Memory Studies», H\&T 41, 2, pp. 179-197.

Klatt, M. 1877: Forschungen zur Geschichte des achäischen Bundes, I. Quellen und Chronologie des Kleomenischen Krieges, Berlín.

Koehn, C. 2007: Krieg-Diplomatie-Ideologie. Zur Aussenpolitik hellenistischer Mittelstaaten, Stuttgart.

Koehn, C. 2013: «Polybios und die Inschriften. Zum Sprachgebrauch des Historikers», en Grieb, V. y Koehn, C. (eds.), Polybios und seine Historien, Stuttgart, pp. 159-181. 
Lafond, Y. 2006 : La mémoire des cités dans la Péloponnèse d'époque romaine (IIe siècle av. J.-C.- IIIe siècle après J.-C.), Rennes.

Larsen, J. 1968: Greek Federal States. Their Institutions and History, Oxford.

Lehmann, G. 2001: Ansätze zu einer Theorie des griechischen Bundesstaates bei Aristoteles und Polybios, Gotinga.

Loraux, N. 2008 [1997]: La ciudad dividida. El olvido en la memoria de Atenas, Buenos Aires.

Lozano, A. 2005: «El problema de la ciudadanía en los estados federales griegos de Época Helenística. El caso de la Confederación Aquea», en Simón, F., Pina Polo, F. y Remesal Rodríguez, J. (eds.), Repúblicas y ciudadanos: modelos de participación cívica en el mundo antiguo, Barcelona, pp. 115-129.

Luraghi, N. 2008: The Ancient Messenians, Cambridge.

Ma, J. 2009: «The City as Memory», en Boys-Stones, G., Graziosi, B. y Vasunia, P. (eds.), The Oxford Handbook of Hellenic Studies, Oxford, pp. 248-259.

Ma, J. 2012: «Honorific Statues and Hellenistic History», en Smith, C. y Yarrow, L. M. (eds.), Imperialism, Cultural Politics, and Polybius, Oxford, pp. 230-251.

Marasco, G. 1981: Commento alle Biografie Plutarchee di Agide e di Cleomene, vol. 2, Roma.

Meadows, A. 2013: «Polybius, Aratus, and the History of the 140th Olympiad», en Gibson, B. y Harrison, Th. (eds.), Polybius and his world. Essays in memory of F. W. Walbank, Oxford, pp. 91-116.

Moreno Leoni, A. 2013: «La unidad del Peloponeso. De la imaginación a la territorialización en la Confederación Aquea helenística durante los siglos III-II a.C.», Polis 25, pp. 99-127.

Mossé, C. 1969: La tyrannie dans la Grèce antique, París.

Nottmeyer, H. 1995: Polybios und das Ende des Achaierbundes. Untersuchungen zu den römisch-achaischen Beziehungen, ausgehend von der Mission des Kallikrates bis zur Zerstörung Korinths, Múnich.

Ober, J. 2003: «Tyrant Killing as Therapeutic Stasis: A political debate in images and texts», en Morgan, K. (ed.), Popular Tyranny. Sovereignity and its Discontents in Ancient Greece, Austin, pp. 215-250.

O’Neil, J. 1984/6: «Political elites in the Achaian and Aitolian leagues», AncSoc 15-17, pp. 33-61.

Orsi, D. 1990: «Il tema antitirannico nella 'Vita di Arato' Plutarchea», GB 17, pp. 147-162.

Orsi, D. 1991: L'Alleanza Acheo-Macedone. Studio su Polibio, Bari.

Page, T. E. 1942: Greek Literary Papyri, vol. 1, Londres.

Pascual, J. 2007: «La sympoliteia griega en las épocas clásica y helenística», Gerión 25, 1, pp. 167-186.

Pédech, P. 1964: La méthode historique de Polybe, París. 
Ricoeur, P. 2004 [2000]: La memoria, la historia, el olvido, Buenos Aires.

Rizakis, A. 1995: Achaie I. Sources Textuelles et Histoire Regionale, París.

Savalli-Lestrade, I. 2009: «Usages civiques et usages dynastiques de la damnatio memoriae dans le monde hellénistique (323-30 av. J.-C.)», en Benoist, St. et al. (eds.), Mémoires partagées, mémoires disputées. Écriture et réécriture de l'histoire, Metz, pp. 127-158.

Scholten, J. 2000: The Politics of Plunder: Aitolians and their Koinon in the Early Hellenistic Era, 279-217 B.C., Berkeley.

Teegarden, D. 2007: Defending Democracy: A Study of Ancient Greek Anti-Tyranny Legislation, $\mathrm{PhD}$ Dissertation, Princeton, mimeo.

Texier, J. G. 1975: Nabis, París.

Tomlinson, R. A. 1972: Argos and the Argolid. From the End of the Bronze Age to the Roman Occupation, Bristol.

Urban, R. 1979: Wachstum und Krise des Achäischen Bundes. Quellenstudien zur Entwicklung des Bundes von 280 bis 222 v. Chr., Wiesbaden.

Vatin, C. 1975: «Polybe, Plutarque et la mort de Machanidas sous les coups de Philopoemen», en Association G. Budé, Actes du IX Congrès, Rome 13-18 avril 1973, París.

Veyne, P. 2005: «L'identité grecque contre et avec Rome», en L'empire grécoromain, París, pp. 205-215.

Walbank, F. 1933: Aratos of Sicyon, Cambridge.

Walbank, F. 1999a [1957]: A Historical Commentary on Polybius, vol. 1, Oxford.

Walbank, F. 1999b [1967], A Historical Commentary on Polybius, vol. 2, Oxford.

Walbank, F. 1999c [1979], A Historical Commentary on Polybius, vol. 3, Oxford.

Walbank, F. 2006a [1984]: «Macedonia and Greece», CAH ${ }^{2}$ VII.1, pp. 219-256.

Walbank, F. 2006b [1984]: «Macedonia and the Greek Leagues», CAH ${ }^{2}$ VII.1, pp. 446-481.

Wilcken, U. 1895: «Aristomachos», RE, II (3), pp. 945-946.

Wolpert, A. 2002: Remembering Defeat. Civil War and Civic Memory in Ancient Athens, Baltimore.

Fecha de recepción de la primera versión del artículo: 28/05/2013

Fecha de aceptación: 18/06/2014

Fecha de recepción de la versión definitiva: 31/07/2014 